\title{
Estenosis bronquial congénita, papel de las imágenes en el diagnóstico: Reporte de un caso
}

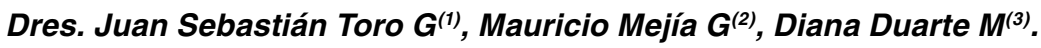

1. Médico Residente Radiología Fundación Valle del Lili-Universidad CES. Cali - Colombia.

2. Medico radiólogo, Fundación Valle del Lili-Universidad CES. Cali - Colombia.

3. Neumóloga Pediatra, Universidad del Bosque, Fundación Valle del Lili. Cali - Colombia.

Congenital bronchial stenosis, the role of imaging in the diagnosis: A case report.

Abstract: This article describes the case of an infant under 2 months old, who at 15 days of age presented stridor and respiratory distress, a chest $X$-ray was performed which reported the presence of a hyperinflation of the right lung field. A fiberoptic bronchoscopy (FBO) was performed which resulted normal and a ventilation/perfusion gammagraph showed a right lung hypoplasia. The infant was treated with additional oxygen and respiratory therapy without clinical. In order to obtain a pre-surgical vascular map, a multislice CT angiography and virtual bronchoscopy were performed, documenting a right bronchial stenosis produced by a fold of the bronchial wall and with valve effect which produced a secondary hyperinsufflation of the right lung. These findings were confirmed in corrective surgery. Keywords (Me SH): Bronchus, CAT, Hypoplasia, Stenosis.

Resumen: En este artículo se reporta el caso de un lactante menor de 2 meses de edad que a los 15 días de nacido presentó estridor y dificultad respiratoria, por lo que consultó a una clínica de primer nivel en donde se le realizó radiografía de tórax, en la cual se documentó la presencia de una hiperinflación del campo pulmonar derecho. Se realizó una fibrobroncoscopía óptica (FBO) que fue reportada como normal y una gammagrafía de ventilación/perfusión que reportó una hipoplasia pulmonar derecha. Fue manejado con oxígeno suplementario y terapias respiratorias sin mejoría clínica, motivo por el cual fue trasladado a nuestra institución para manejo especializado. Con el fin de obtener un mapa vascular pre quirúrgico, se realizó una angiografía pulmonar por tomografía (Figura 2) reconstrucciones con mínima intensidad de proyección (Figura 3) y broncoscopía virtual (BV) multicorte (Figuras 4 y 5) documentándose una estenosis del bronquio fuente derecho, producida por un repliegue de la pared bronquial y con efecto de válvula que producía una hiperinsuflación secundaria del pulmón derecho. Estos hallazgos fueron confirmados en la cirugía correctiva.

Palabras clave (DeCS): Bronquios, Estenosis, Hipoplasia, TAC.

Toro JS, et al. Estenosis bronquial congénita, papel de las imágenes en el diagnóstico: Reporte de un caso. Rev Chil Radiol 2014; 20(2): 64-67.

Correspondencia: Juan Sebastián Toro Gutiérrez / juansetoro85@hotmail.com

Trabajo recibido el 21 de agosto de 2013. Aceptado para publicación el 22 de mayo de 2014.

\section{Introducción}

El desarrollo y crecimiento de la vía aérea comienza en la cuarta semana de gestación y continúa hasta los 2 años de edad. Este desarrollo se puede definir en 5 fases secuenciales: fase embrionaria, en la que se forman las yemas pulmonares y el septo traqueo esofágico; fase pseudoglandular, en la cual se forman los bronquios; fase canalicular, en la cual se organiza la red capilar y acinos; fase sacular, encargada de formar los septos alveolares e intersticio; y por último, la fase alveolar en la cual se terminan de madurar los alveolos primitivos ${ }^{(1,2)}$. Una alteración en cualquiera de estas fases conlleva a la aparición de anomalías congénitas pulmonares ${ }^{(2)}$. La estenosis bronquial se produce específicamente por una alteración en la fase pseudoglandular y generalmente se acompaña de otras malformaciones congénitas, ya sean cardiacas, esofágicas y del esqueleto, sin embargo, también puede encontrarse de manera aislada ${ }^{(3)}$.

En la actualidad la incidencia anual estimada de 
malformaciones pulmonares congénitas es de 30 a 42 casos por 100.000 personas. Las más frecuentes son la traqueobroncomalacia, las malformaciones congénitas pulmonares de la vía aérea (antes denominadas malformaciones adenomatoideas quísticas), el enfisema lobar congénito, el secuestro pulmonar, el síndrome de cimitarra, la atresia bronquial, el bronquio traqueal, los quistes broncogénicos y la estenosis traqueobronquial(4).

La estenosis traqueobronquial congénita se define como una disminución fija en el calibre de la tráquea o de un bronquio debido a una alteración en la en la etapa fetal, y cuya incidencia es desconocida ${ }^{(5)}$. Los sitios de mayor compromiso son principalmente la tráquea en su tercio inferior o los bronquios principales, pudiendo ser producidos por causas extrínsecas o intrínsecas. El $50 \%$ de las estenosis traqueobronquiales son focales, $30 \%$ difusas y $20 \%$ en forma de embudo ${ }^{(1,5)}$. En un estudio realizado en el 2007 por Heyer y cols se demostró que las causas extrínsecas más frecuentes de estenosis traqueobronquial son las malformaciones vasculares, dentro de las cuales las más frecuentes son la presencia de un tronco braquiocefálico inmediatamente anterior a la tráquea, doble arco aórtico, anillo vascular y arteria subclavia derecha aberrante; la causa intrínseca más frecuente fue la traqueobroncomalacia(6).

En este artículo se reportan las características histopatológicas, imagenológicas y quirúrgicas de un caso de estenosis intrínseca del bronquio derecho secundario a la presencia de un repliegue cartilaginoso que estrechaba la luz del bronquio principal, produciendo un fenómeno de válvula con atrapamiento de aire distal. El caso adquiere interés debido a lo infrecuente de la patología y al papel fundamental jugado por la radiología en el diagnóstico no invasivo del paciente.

\section{Reporte de caso}

El paciente es un lactante menor gemelar de 2 meses de edad sin antecedentes prenatales de importancia. Ingresó al servicio de urgencias de nuestra institución proveniente de otro centro por un cuadro clínico de un mes y medio de evolución, consistente en estridor y disnea. En ese centro se realizaron varios exámenes entre ellos una radiografía de tórax que demostró una hiperinsuflación del campo pulmonar derecho sin otros hallazgos, una fibrobroncoscopía óptica (FBO) la cual fue interpretada como normal y una gammagrafía de ventilación/ perfusión que reportó una hipoplasia pulmonar derecha, por lo cual se estableció un plan terapéutico inicial basado en lobectomía pulmonar ipsilateral.

Debido al deterioro clínico, el paciente fue remitido a nuestra institución donde se decidió complementar el diagnóstico con la realización de una nueva radiografía de tórax que confirmó la hiperinsuflación pulmonar derecha con desviación del cardiomediastino hacia la izquierda (Figura 1), sin masas o lesiones hiliares. Basados en estos hallazgos se recomendó la realización de TAC multicorte con reconstrucciones multiplanares en fase angiográfica pulmonar para evaluar un posible anillo vascular y una broncoscopía virtual para descartar lesión intrínseca de la vía aérea.

Este examen mostró una asimetría en el volumen de ambos campos pulmonares por hiperinsuflación del pulmón derecho secundario a una estenosis bronquial derecha significativa condicionada por un repliegue de tejidos blandos localizado en el sitio de origen de este bronquio, con efecto de válvula asociado (Figura 2). No se identificaron causas extrínsecas que explicaran la estenosis y el parénquima pulmonar no mostró formaciones quísticas ni alteraciones en las estructuras vasculares pulmonares o sistémicas. No había signos de hipoplasia pulmonar.

El paciente fue llevado a cirugía con el diagnóstico imagenológico de una estenosis intrínseca proximal del bronquio fuente derecho, en donde se confirmó la presencia de un repliegue dependiente de la pared posterior de este bronquio que fue resecado, con anastomosis bronquial término-terminal. La histopatología confirmó la presencia de un tejido cartilaginoso como causa de la estenosis bronquial. El paciente egresó de la institución con una mejoría completa de sus síntomas y persistió asintomático en sus controles posteriores.

\section{Discusión}

La radiografía convencional juega un papel en la detección imagenológica inicial en pacientes con sospecha clínica de anomalías pulmonares congénitas. Sin embargo, en recientes años hay un aumento en el uso de la tomografía computarizada (TAC), pues no sólo permite confirmar el diagnóstico, sino a su vez caracterizar el tipo de anomalía, planear la corrección quirúrgica en casos en los que se necesite y valorar las anomalías vasculares asociadas ${ }^{(4,7)}$. Recientemente, con el desarrollo y la amplia disponibilidad de la TAC multidetector, esta modalidad ha asumido un mayor papel en la evaluación no invasiva de las anomalías congénitas pulmonares gracias a la combinación de la alta velocidad de adquisición, alta resolución espacial, mejor calidad de reconstrucción multiplanar (MPR), reconstrucciones en tres dimensiones (3D) y la capacidad de realizar broncoscopía virtual (BV) $)^{(7)}$.

La fibrobroncoscopía óptica (FBO) es la mejor modalidad para la evaluación de lesiones endoluminales y de la mucosa de las vías respiratorias ${ }^{(8)}$. La FBO generalmente aporta una triple función. En primer lugar, provee una guía exploratoria permitiendo la visualización anatómica y funcional de la vía aérea con la opción de realizar diagnósticos mediante la toma de muestras de tejido, la realización de lavado broncoalveolar y la valoración dinámica en 


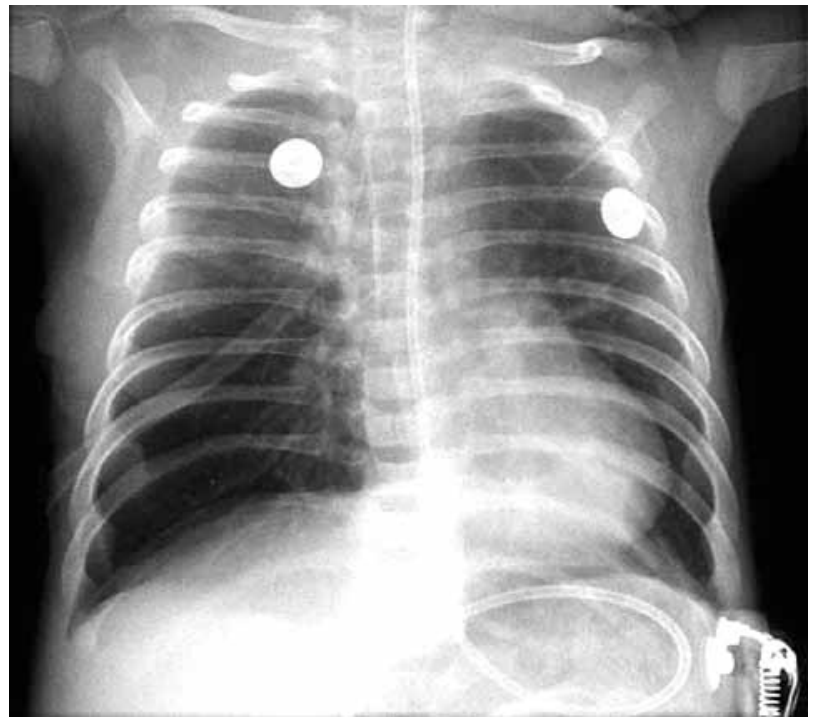

Figura 1. Rx tórax ap. Aumento de la radiolucidez y del volumen del hemitórax derecho con desplazamiento del cardiomediastino hacia el lado contralateral sin lesiones hiliares.

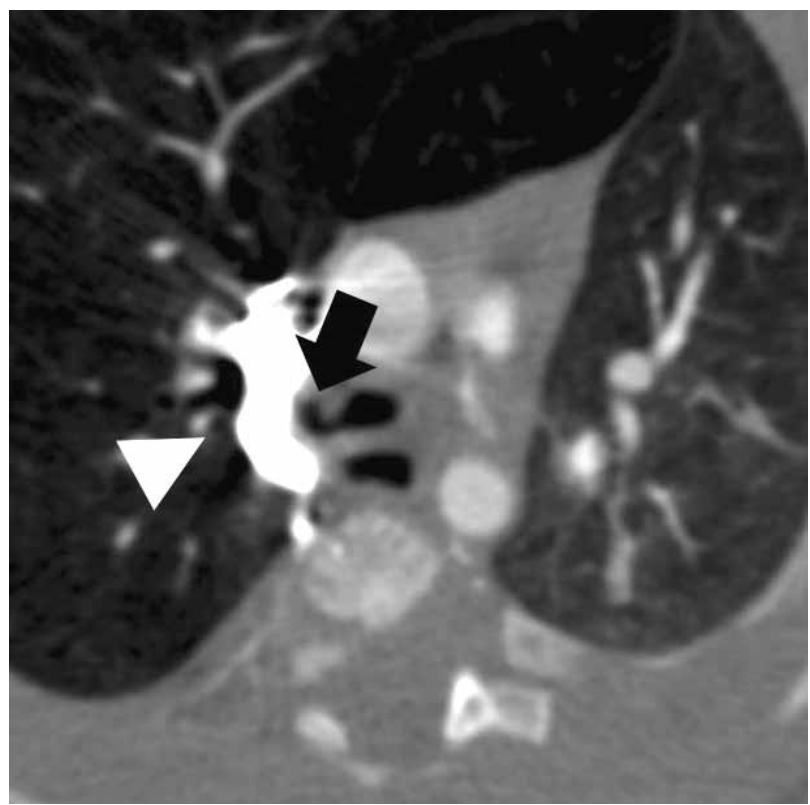

Figura 2. TAC de tórax corte a nivel de carina. Se identifica estenosis de casi toda la luz del bronquio principal derecho producida por un repliegue anterior originado de la pared bronquial (flecha negra). No se observan causas extrínsecas de la estenosis. Medio de contraste no diluido en la vena ácigos (cabeza de flecha).

inspiración-espiración de la vía aérea. Esta última función fue determinante en la causa de falsos negativos reportadas en la TAC multidetector con BV, que terminaron en traqueobroncomalacia, según el estudio hecho por Heyes y cols ${ }^{(6)}$. Adicionalmente, el

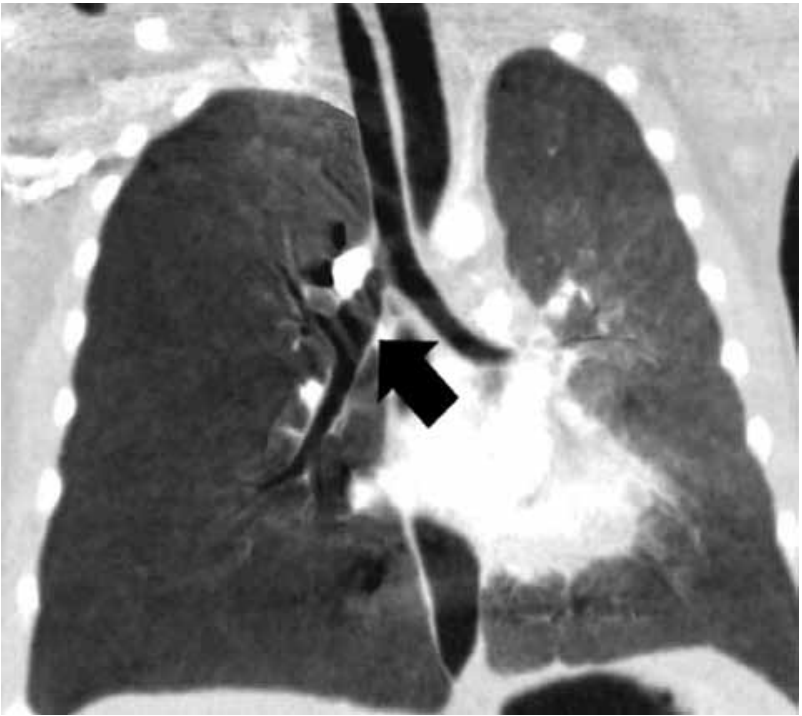

Figura 3. Reconstrucción multiplanar fina con algoritmo MinMIP. Hay disminución en el calibre del bronquio principal derecho con efecto de válvula que condiciona a aumento en el volumen del pulmón derecho comparado con el contralateral.

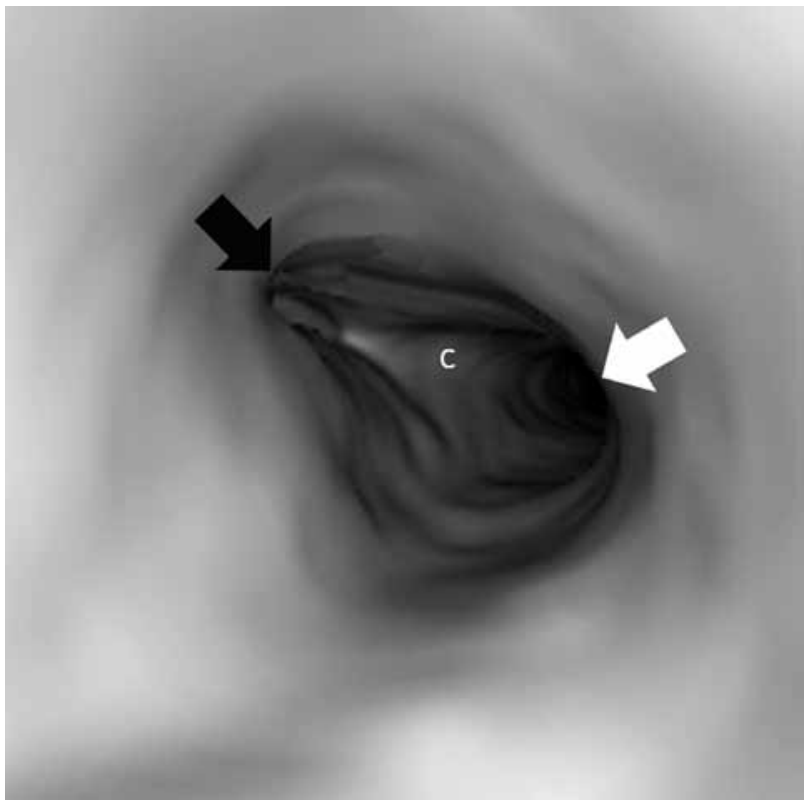

Figura 4. Broncoscopía virtual (BV) a nivel del tercio distal de la tráquea. Inmediatamente luego de la carina $(C)$ se observa la estenosis del bronquio derecho (flecha negra).El bronquio izquierdo (flecha blanca) no muestra alteraciones.

procedimiento permite realizar intervenciones terapéuticas, por medio de la aspiración de secreciones y cuerpos extraños. En los pacientes con sospecha de una malformación pulmonar congénita, la FBO es una prueba útil y puede llevar a un diagnóstico 


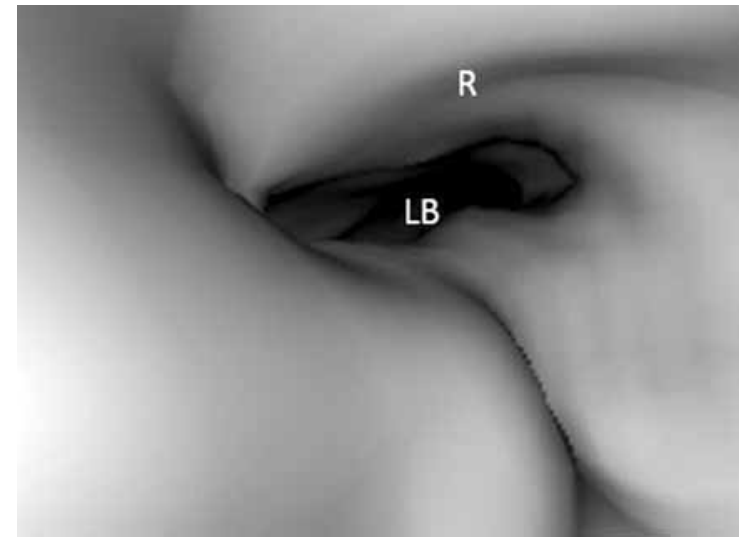

Figura 5. Broncoscopía virtual (BV) a nivel del bronquio fuente derecho. Importante disminución de la luz bronquial $(L B)$ por presencia de un repliegue $(R)$ de morfología en espiral en la pared bronquial anterior que produce efecto de válvula.

definitivo. Sin embargo, es frecuente que se requieran pruebas complementarias, tal como lo demostró un estudio reciente hecho por Wang et a/(6), donde la FBO mostró una sensibilidad del $50 \%$ en pacientes con atresia traqueobronquial requiriendo en todos los casos estudios complementarios como el CT de tórax para su diagnóstico. La FBO tiene limitaciones importantes debido a que no es posible valorar estructuras por fuera de la pared bronquial, tales como los ganglios linfáticos mediastinales o las estructuras vasculares, $y$ frecuentemente proporciona poca información sobre la magnitud del compromiso extraluminal o sobre las características de la vía aérea distal en una estenosis de alto grado bronquial ${ }^{(8)}$. En vista de estas limitaciones, los dos exámenes pueden ser complementarios, esta afirmación puede considerarse teniendo en cuenta la alta sensibilidad $(87 \%)$, especificidad ( $86 \%$ ) y valor predictivo positivo (97\%) de la TAC multidector con BV(5).

La BV se puede realizar cuando FBO está contraindicada o cuando sea riesgosa, como en los casos de estrechez severa de las vías respiratorias permitiendo una evaluación no invasiva intraluminal del árbol traqueobronquial y proporcionando información sobre el compromiso extraluminal ${ }^{(5)}$.

Es difícil explicar los resultados equívocos de la FBO inicial, sin embargo las causas más frecuentes de estos resultados son la poca experiencia de este procedimiento en la población pediátrica. En nuestra institución, a pesar de tener una amplia experiencia en esta población, no se realizó la FBO debido a los hallazgos contundentes del TAC multidector con
BV, que permitió dar un diagnóstico rápido y certero debido a su gran resolución espacial, con técnicas de reconstrucción multiplanar y de navegación endolumninal, además de permitir una adecuada planeación pre quirúrgica en un caso de estenosis bronquial congénita.

\section{Conclusión}

Los pacientes con sospecha de malformaciones traqueo-bronquiales o pulmonares, pueden beneficiarse de la Tomografía Multicorte con reconstrucciones endoluminales del árbol traqueo-bronquial (broncoscopía virtual), como herramienta no invasiva que complemente los hallazgos obtenidos con una fibrobroncoscopía óptica. Este caso ilustra como el método imagenológico pudo orientar el diagnóstico de una estenosis bronquial congénita en un lactante con un pulmón hiperinsuflado y estridor.

\section{Bibliografía}

1. Berrocal T, Madrid C, Novo S, Gutiérrez J, Arjonilla A, Gómez-León N. Congenital Anomalies of the Tracheobronchial Tree, Lung, and Mediastinum: Embryology, Radiology, and Pathology. RadioGraphics 2004; 24: e17- January.

2. Deepa R, Chapman T, Ferguson M, Deutsch G, Manjiri D. Congenital Lung Abnormalities: Embryologic Features, Prenatal Diagnosis, and Postnatal Radiologic-Pathologic Correlation. Radiographics 2010; 30: 1721-1738.

3. Jaffe R. Balloon Dilation of Congenital and Acquired Stenosis of the Trachea and Bronchi. Radiology 1997; 203: 405-409.

4. Lee E, Dorkin H, Vargas S. Congenital Pulmonary Malformations in Pediatric Patients: Review and Update on Etiology, Classification, and Imaging Findings. Radiol Clin N Am 2011; 49: 921-948.

5. Lee E, Bruce, Phillip B. Multidetector computed tomography of pediatric large airway diseases: State-of-theArt. Radiol Clin N Am 2011; 49: 869-893.

6. Heyer C, Nuesslein T, Jung D, Soeren P, Lemburg $\mathrm{S}$, Rieger $\mathrm{C}$ et al. Tracheobronchial Anomalies and Stenoses: Detection with Low-Dose Multidetector CT with Virtual Tracheobronchoscopy Comparison with Flexible Tracheobronchoscopy. Radiology 2007; 242: 2-February.

7. Lee E, Phillip M, Boisell H. Multidetector CT Evaluation of Congenital Lung Anomalies. Radiology 2008; 247: 3-June.

8. Saad H. Role of Virtual Bronchoscopy in the Evaluation of Bronchial Lesions: A Pictorial Essay. Current Problems in Diagnostic. Radiology 2013 ; 42: 33-39.

9. Wang Y, Weimin D, Yu'e S, Xiangyang C, Yang B, Zhao M. Congenital Bronchial Atresia: Diagnosis and Treatment. International Journal of Medical Sciences 2012; 9: 207-212. 